\title{
Mapa de preferência de sorvetes ricos em fibras
}

\author{
Preference map of high-fiber ice cream
}

\author{
Regiane Lopes de SALES ${ }^{1}$, Ana Carolina Pinheiro VOLP ${ }^{1}$, Kiriaque Barra Ferreira BARBOSA ${ }^{1}$, \\ Maria Inês de Souza DANTAS ${ }^{1}$, Hércia Stampini DUARTE², Valéria Paula Rodrigues MINIM ${ }^{1 *}$
}

\section{Resumo}

Quatro amostras de sorvete foram preparadas adicionando-se diferentes concentrações de granola e Frutooligossacarídeo (FOS). Foi avaliado o teor de fibras segundo método AOAC. A avaliação da aceitabilidade sensorial foi realizada por 78 consumidores, com idade entre 15 e 25 anos. Estes avaliaram a aceitação global das formulações, utilizando a escala hedônica de nove pontos, em uma única seção, de forma monádica. O experimento foi estruturado segundo delineamento em blocos inteiramente casualizados. Os resultados foram submetidos à análise de variância, ao teste de médias de Tuckey e à metodologia do Mapa de Preferência interno para análise dos componentes principais. As amostras controle e as adicionadas de FOS tiveram melhor aceitação. A amostra contendo somente FOS foi a que mais se aproximou do produto tradicional, não diferindo significativamente quanto à aceitação global. A granola distinguiu-se estatisticamente do produto tradicional. O teor de fibras analisado foi superior ao fornecido pelo fabricante da granola. As concentrações de fibras nos sorvetes variaram de 0,9 a 5,45 g. $100 \mathrm{~g}^{-1}$ de sorvete, com a maior concentração de fibras provenientes do FOS. Esse resultado permite concluir que o FOS é um ingrediente promissor no desenvolvimento de produtos ricos em fibras.

Palavras-chave: aceitação sensorial; análise de componentes principais; Mapa de Preferência interno; sorvete.

\begin{abstract}
Four samples of ice cream were prepared with four different concentrations of granola and fructooligosaccharide (FOS). The concentration of fiber was evaluated using the AOAC method. The acceptance evaluation was conducted by 78 consumers between 15 and 25 years of age. The consumers evaluated the global acceptance of the formulations through the structured 9-point hedonic scale, in a single section, in a monadic form. The experiment was structured by a Randomized Complete Block Design. The results were submitted to the analysis of variance, Tukey test, and to the preference mapping technique for principal component analysis. The control samples and the others with FOS had the better acceptance. The sample containing solely FOS was the closest to the traditional product with little difference from the latter in terms of global acceptance. The granola was statistically different from the traditional product. The fiber concentration analyzed was higher than the one provided by the granola supplier. The fiber concentrations in the ice creams varied from 0,9 to $5,45 \mathrm{~g} .100 \mathrm{~g}^{-1}$. The highest concentration was the one from the FOS. This result shows that FOS is a promising ingredient in the development of fiber-rich products. Keywords: sensorial acceptance; principal components analysis; internal preference mapping; ice cream.
\end{abstract}

\section{Introdução}

O avanço dos conhecimentos sobre a relação entre alimentação e saúde, bem como os elevados custos da saúde pública e a busca permanente da indústria por inovações têm gerado novos produtos, cujas funções pretendem ir além do conhecido papel nutricional dos alimentos. Pesquisas têm sido realizadas visando à identificação de novos compostos bioativos e o estabelecimento de bases científicas para a comprovação das alegações de propriedades funcionais dos alimentos.

O mercado para esse tipo de alimento movimenta cerca de US\$ 60 bilhões no mundo, responsável por mais da metade dos investimentos publicitários na área alimentícia e com expectativas de crescimento da ordem de 5\% ao ano (HARDY, 2000; SWADLING, 2001). Trata-se, portanto, de um segmento de grande interesse para as indústrias alimentícias e farmacêuticas, que têm buscado explorar a relação entre o consumo de determinados ingredientes com a redução de fatores de risco para doenças específicas, principalmente as crônicas não transmissíveis, ou a melhora do desempenho físico ou mental (GRIZARD; DALLE; BARTHOMEUF, 2001; IZZO; NINESS, 2001).

As fibras insolúveis produzem redução no tempo de trânsito intestinal e aumento do volume fecal levando à diminuição da constipação intestinal, e as fibras solúveis provocam retardo no esvaziamento gástrico, na absorção da glicose e do colesterol, permitindo melhor controle dos níveis plasmáticos, promovendo redução do risco de doenças cardiovasculares e diabetes, além de contribuir com a saciedade (COZZOLINO, 2005; CUMMINGS; EDMOND; MAGEE, 2004).

Dentre os alimentos ricos em fibras, destaca-se a granola, que é um produto alimentar constituído por uma mistura de frutas secas, grãos de cereais e sementes oleaginosas, tais como amendoim e castanha-do-pará (GRANADA et al., 2003). Tal

Recebido para publicação em 8/5/2008

Aceito para publicação em 17/7/2008 (002337)

${ }^{1}$ Departamento de Engenharia de Alimentos, Universidade Federal de Viçosa - UFV, Campus Universitário, CEP 36570-000, Viçosa - MG, Brasil, E-mail: vprm@ufv.br

${ }^{2}$ Departamento de Nutrição e Saúde, Universidade Federal de Viçosa - UFV, Viçosa - MG, Brasil

${ }^{*}$ A quem a correspondencia deve ser enviada 
produto tem em seu teor de fibras a predominância da fibra insolúvel.

Em relação às fibras solúveis, os Frutooligossacarídeos (FOS) têm despertado grande interesse. Constituídos de moléculas de sacarose, nas quais uma ou duas unidades de frutose são adicionadas por ligações $\beta$-(2-1). Os derivados de sacarose são encontrados naturalmente em vegetais e plantas como a alcachofra, a raiz de chicória, o alho, a cebola, a banana, dentre outras, embora em quantidades pequenas, exigindo consumo elevado para se obter o efeito funcional esperado (FERREIRA, 2001).

Entre as propriedades prebióticas, existe um consenso de que os FOS modificam o habitat intestinal, causando aumento no volume fecal e normalização da freqüência fecal; aumentando a proliferação de bactérias e/ou a atividade do número de bifidobactérias e bactérias ácido lácticas no intestino humano (GERMAN et al., 1999). Segundo Rodríguez et al. (2003), os FOS podem também exercer efeitos benéficos na prevenção e no tratamento de doenças crônicas não transmissíveis.

O sucesso do alimento no mercado depende de seu desempenho junto ao consumidor. A determinação da aceitação e/ou preferência do produto se torna indispensável no processo de desenvolvimento de novos produtos, bem como no melhoramento de processos e na substituição de ingredientes (RODRÍGUEZ; MEGÍAS; BAENA, 2003).

As metodologias tradicionais para analisar dados de testes afetivos têm mostrado limitações e deficiências. Geralmente após a realização dos testes afetivos, os dados são analisados estatisticamente por meio da análise de variância e por testes de comparação de médias. Dessa forma, para cada produto avaliado obtém-se a média do grupo de consumidores assumindo, portanto, que todos os respondentes possuem o mesmo comportamento, desconsiderando suas individualidades. Com isto, pode estar ocorrendo perda de importantes informações (REIS et al., 2006).

Com a finalidade de analisar os dados afetivos, levando-se em consideração a resposta individual de cada consumidor, e não somente a média do grupo de consumidores que avaliaram os produtos, foi desenvolvida a técnica intitulada Mapa de Preferência, que tem sido largamente utilizada por cientistas da área de análise sensorial (BEHRENS; SILVA; WAKELING, 1999).

Com base no exposto, o objetivo do presente estudo foi desenvolver um novo produto e avaliar o efeito da adição de diferentes concentrações de fibra e/ou prebiótico (Frutooligossacarídeos (FOS)) na sua aceitação, utilizando a técnica do Mapa de Preferência para análise dos resultados.

\section{Material e métodos}

\subsection{Material}

Quatro amostras de sorvete de creme (marca comercial) foram preparadas, adicionando-se diferentes concentrações de fibra e prebiótico. Como fonte de fibra utilizou-se granola obtida no comércio local e o prebiótico Frutooligossacarídeo (FOS) (Nutraflora ${ }^{\oplus}$ 95, Corn Products Brasil).

\subsection{Métodos}

\section{Preparo das amostras}

As amostras foram preparadas no Laboratório de Estudo Experimental dos Alimentos do Departamento de Nutrição e Saúde da Universidade Federal de Viçosa. A quantidade dos ingredientes adicionados às amostras está apresentada na Tabela 1. As porcentagens adicionadas foram calculadas sobre a massa total do sorvete.

\section{Análise de fibra alimentar}

As amostras foram avaliadas, em duplicata, quanto ao teor de Fibra Alimentar Total (FAT) e Insolúvel (FAI) utilizando-se o método enzimático gravimétrico, preconizado pela Association of Official Analytical Chemists (2002). A Fibra Alimentar Solúvel (FAS) foi obtida por diferença entre FAT e FAI.

\section{Análise sensorial}

A avaliação da aceitabilidade sensorial das amostras foi realizada por 78 consumidores. Os consumidores avaliaram a aceitação global das formulações, utilizando a escala hedônica de nove pontos (REIS; MINIM, 2006). O teste foi realizado em uma sorveteria central da cidade de Viçosa-MG, onde as amostras de sorvete foram avaliadas, na mesma seção, por cada consumidor. As amostras foram servidas de forma monádica, em copos descartáveis de $50 \mathrm{~mL}$, codificados com números aleatórios de três dígitos.

O experimento foi estruturado segundo o delineamento em blocos completos casualizados.

\section{Análise estatística}

Os dados referentes à aceitação das quatro amostras foram primeiramente submetidos a uma análise de variância (ANOVA) e ao teste de Tukey a 5\% de probabilidade, tendo-se como fontes de variação amostras e provadores.

Para obtenção do Mapa de Preferência Interno ou Análise de Preferência Multidimensional (MDPREF), os dados de aceitação (teste de consumidor) foram organizados numa matriz de amostras (em linhas) e consumidores (em colunas), e esta submetida à Análise de Componentes Principais (ACP) (REIS et al., 2006).

Os resultados foram expressos em um gráfico de dispersão das amostras (tratamentos) em relação aos dois primeiros componentes principais e em outro representando os loadings (car-

Tabela 1. Ingredientes adicionados às amostras de sorvete (g. $\left.100 \mathrm{~g}^{-1}\right)$.

\begin{tabular}{lcccc}
\hline Ingredientes & \multicolumn{4}{c}{ Amostras } \\
\cline { 2 - 5 } & Sorvete FOS & $\begin{array}{c}\text { Sorvete } \\
\text { GRA }\end{array}$ & $\begin{array}{c}\text { Sorvete } \\
\text { FOS + GRA }\end{array}$ & Controle \\
\hline FOS & 5,0 & 0 & 5,0 & - \\
Granola & 0 & 10,0 & 5,0 & - \\
\hline
\end{tabular}

FOS = frutooligossacarídeo; e GRA = granola. 
gas) da ACP (correlações dos dados de cada consumidor com os dois primeiros componentes principais) (REIS et al., 2006).

A análise estatística foi realizada utilizando-se o software Statistical Analysis Systems (SAS) versão 8,0, licenciado pela Universidade Federal de Viçosa.

\section{Resultados e discussão}

\subsection{Fibra alimentar}

A amostra de granola analisada segundo o método AOAC (2002) apresentou um teor de $8,97 \%$ de fibra alimentar total, sendo $60 \%$ correspondente à fibra insolúvel $(5,34 \mathrm{~g})$ e $40 \%$ à solúvel (3,63 g).

A quantidade de fibra especificada na embalagem da granola utilizada foi menor em relação à analisada, 6,5\% contra 8,97\%. Cabe ressaltar, que o fabricante não discrimina a porcentagem de fibra solúvel e insolúvel, disponibilizando somente o teor de fibra alimentar total. Nenhuma informação foi fornecida em relação ao método utilizado para a análise de fibras em tal produto.

Segundo Ribeiro et al. (2003), os dados de composição química dos alimentos consistem em uma importante limitação, uma vez que se trata de um processo complexo, fortemente influenciado pela qualidade das informações disponíveis. Entre os principais fatores que podem resultar na imprecisão dos dados disponíveis, destacam-se: a descrição incorreta de alimentos e/ou fontes de valores nutricionais; a amostragem inadequada; a utilização de métodos analíticos impróprios; a inconsistência na terminologia utilizada para expressar certos nutrientes e a variabilidade resultante de fatores genéticos, ambientais e de preparo e processamento dos alimentos.

Dentre as amostras testadas, a amostra contendo FOS + granola foi a que se constituiu na melhor fonte de fibras, apresentando 5,45 g de fibra por $100 \mathrm{~g}$ de sorvete (Tabela 2).

Conforme dados da tabela para Avaliação do Consumo Alimentar em Medidas Caseiras (PINHEIRO et al., 2005), o consumo de $160 \mathrm{~g}$ de sorvete (FOS + granola), porção usualmente consumida, equivalente a duas bolas, resulta na ingestão de $8,72 \mathrm{~g}$ de fibras, o que implica no fornecimento de cerca de $30 \%$ do valor da Ingestão Adequada (AI) de fibras para um indivíduo adulto (NATIONAL ACADEMY OF SCIENCES, 2002).

O teor de fibras presente nos sorvetes desenvolvidos chama a atenção, principalmente na amostra referente à FOS + granola, uma vez que tal teor supera ou se aproxima ao encontrado em alimentos considerados como fontes de fibras características na alimentação. Entre tais alimentos destacam-se barra de cereais,

Tabela 2. Teor de fibra alimentar adicionada às amostras de sorvete (g.100 g-1).

\begin{tabular}{ccccc}
\hline Ingredientes & \multicolumn{4}{c}{ Amostras } \\
\cline { 2 - 5 } & Sorvete FOS & $\begin{array}{c}\text { Sorvete } \\
\text { GRA }\end{array}$ & $\begin{array}{c}\text { Sorvete } \\
\text { FOS + GRA }\end{array}$ & Controle \\
\hline FOS & 5,0 & 0 & 5,0 & - \\
Granola & 0 & 0,9 & 0,45 & - \\
Total & 5,0 & 0,9 & 5,45 & 0 \\
\hline FOS = frutooligossacarídeo e GRA $=$ grano & & &
\end{tabular}

FOS = frutooligossacarídeo; e GRA = granola arroz integral, pão integral, aveia e feijão, entre outros, cujos teores de fibra alimentar total variam de 4,0 a $6,8 \mathrm{~g}$ por $100 \mathrm{~g}$ de produto (FRANCO, 1999).

A adoção de dieta rica em fibras tem sido associada a diversos benefícios para a saúde humana, tais como, baixas concentrações de lipídios séricos e baixos níveis de adiposidade corporal e ainda, baixa incidência de mortes por isquemia do miocárdio, diabetes mellitus e certos tipos de câncer, além de uma maior expectativa de vida (FERREIRA; BURINI; MAIA, 2006; QI et al., 2006; RODRÍGUEZ et al., 2006; SARTORELLI; FRANCO, 2003).

\subsection{Aceitabilidade sensorial}

As médias obtidas para cada tratamento estão apresentadas na Tabela 3, onde se observa que a maior média de julgamentos foi para o sorvete adicionado de FOS, que não diferiu estatisticamente das amostras controle e contendo FOS + granola. No entanto, o sorvete contendo somente granola apresentou menor média de julgamentos, diferindo significativamente do sorvete contendo FOS.

Com os dados obtidos no teste de aceitação das quatro amostras de sorvete com fibras, foi realizada a análise do Mapa de Preferência interno (Figuras 1 e 2). O primeiro Componente Principal (PC) explicou 54\% e o segundo, 33\%. Os dois primeiros componentes principais explicam a maior parte de variância (87\%) entre as amostras quanto à sua aceitação. Assim, apenas os dois primeiros componentes principais são suficientes para discriminar as amostras quanto à aceitação.

Tabela 3. Média de julgamento para diferentes amostras de sorvete.

\begin{tabular}{lc}
\hline Amostra & Média \\
\hline FOS & $7,82^{\mathrm{a}}$ \\
Controle & $7,53^{\mathrm{ab}}$ \\
FOS + GRA & $7,41^{\mathrm{ab}}$ \\
GRA & $7,23^{\mathrm{b}}$
\end{tabular}

FOS = frutooligossacarídeo; e GRA = granola. As médias seguidas de letras distintas diferem entre si pelo teste de Tukey a $5 \%$ de probabilidade.

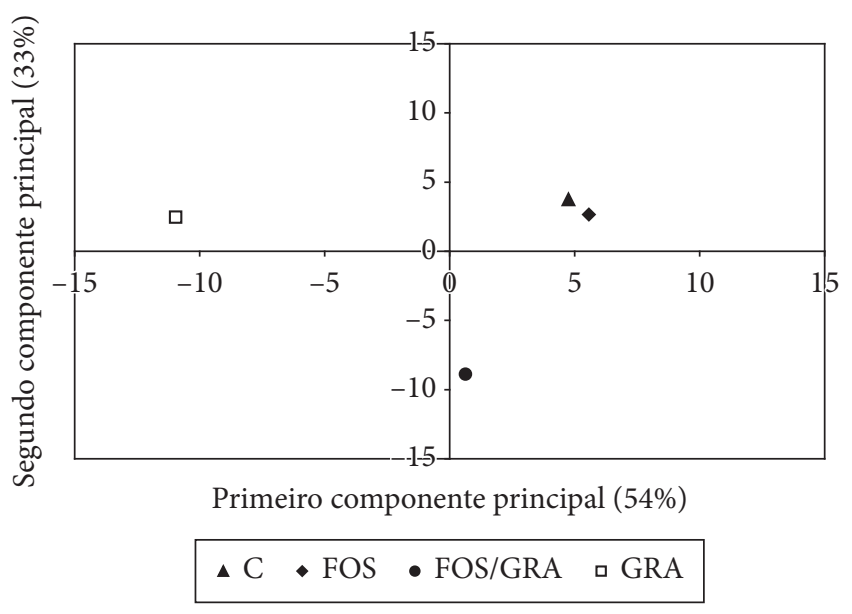

Figura 1. Dispersão das amostras de sorvete em relação à aceitação pelos consumidores. 


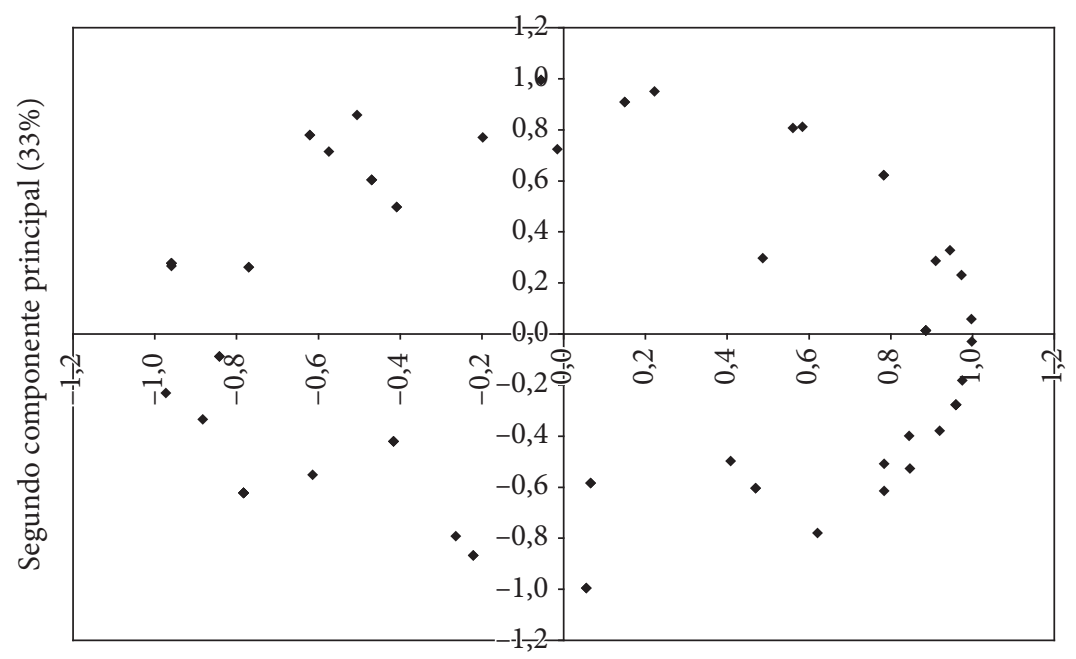

Primeiro componente principal (54\%)

Figura 2. Loadings (cargas). Correlações entre os dados de aceitação de cada consumidor e os dois primeiros componentes principais.

A separação espacial das amostras de sorvete sugere a existência de três grupos de acordo com a aceitação das mesmas, sendo um grupo formado pelas amostras controle e FOS e os outros pelas amostras com FOS/granola e granola. Assim, pode-se sugerir que a presença de granola no sorvete alterou as características do produto, formando grupos distintos do controle e FOS (Figura 1).

Na Figura 2, cada ponto representa as correlações entre os dados de aceitação de um consumidor e os dois primeiros componentes principais. Os consumidores correlacionados com pelo menos um dos componentes consideram diferença na aceitação das amostras. A maioria dos consumidores se correlacionara positivamente com o primeiro componente principal, indicando que atribuíram notas mais elevadas para as amostras.

A maior aceitação do sorvete contendo FOS e granola, em relação à amostra contendo somente granola, pode ser atribuída a dois fatores, primeiro pela menor quantidade de granola na amostra contendo FOS (5\%) e segundo a maior doçura, já que o FOS possui também um poder edulcorante.

Observando-se as médias de julgamentos para as diferentes amostras (Tabela 3), é possível inferir que todas as amostras, independente do tipo e da concentração de fibras adicionada, constituem-se boas alternativas de mercado, uma vez que apresentaram médias de julgamentos entre 7 e 8 (escala de 9 pontos). Tal fato é importante, já que se trata de um produto de boa aceitação no mercado, ao qual foi agregado valor nutricional, adicionando fibras.

O FOS, por não alterar as características do produto, pode ser adicionado ao sorvete convencional sem perdas na qualidade do mesmo e com ganho para contribuir ao equilíbrio da microbiologia intestinal (SGARBIERI; PACHECO, 1999; URGELL; ORLEÁNS; SEUMA, 2005). Segundo a ANVISA (2008), 1,5 g de FOS em uma porção diária de alimento líquido já permitiria a alegação de alimento com propriedade funcional. A melhor formulação testada apresentou $5 \mathrm{~g}$ de FOS. $100 \mathrm{~g}^{-1}$ de sorvete, valor superior ao estabelecido. Entretanto, segundo informe técnico $\mathrm{n}^{\circ}$ 9, de 21 de maio de 2004 (ANVISA, 2008), a alegação de propriedades funcionais para nutrientes com função plenamente conhecida pela comunidade não pode ser aplicada ao sorvete, por este ser um alimento de consumo ocasional (RDC n 359/03) (ANVISA, 2008).

Para aprovação da alegação funcional do sorvete com FOS novos estudos devem ser realizados para provar a sua funcionalidade.

Diante da sua capacidade em aumentar a viscosidade intestinal e favorecer resistência ao fluxo do processo digestivo, podendo impedir a digestão e a absorção de nutrientes no intestino, o FOS pode exercer efeitos benéficos na prevenção e no tratamento de doenças crônicas não transmissíveis, entre elas a obesidade e a diabetes (RODRÍGUEZ; MEGÍAS; BAENA, 2003).

Acredita-se que outros mecanismos possam estar envolvidos na redução de colesterol sangüíneo pelas propriedades funcionais do FOS. Dentre esses, a redução do colesterol plasmático. Quando metabolizado pelas bactérias colônicas, produz ácidos graxos de cadeia curta: acetato, propionato e butirato. Os dois primeiros podem ser absorvidos e empregados para obtenção de energia. O propionato possui uma ação inibidora sobre a hidroxi-metilglutaril coenzima A redutase (HMG CoA redutase), enzima responsável pela síntese do colesterol endógeno (RODRÍGUEZ; MEGÍAS; BAENA, 2003; RODRÍGUEZ et al., 2006).

Já a adição de granola ao sorvete vem de encontro a outro grupo de consumidores, distinto dos primeiros, que buscam alimentos integrais, constituindo assim uma alternativa de mercado, colaborando com a ingestão de fibra insolúvel.

A fibra insolúvel também desempenha ações funcionais que se atribuem ao incremento do volume fecal; estímulo da mobilidade intestinal, freqüência das defecações; aumento da excreção de ácidos biliares e propriedades antioxidantes e hipocolesterolêmicas (GUILLON; CHAMP, 2000). Rodriguez et al. (2003) acrescenta que a fibra insolúvel também contribui para a maior necessidade de mastigação, relevante nas sociedades mo- 
dernas vítimas da ingestão compulsiva e da obesidade. Estudos epidemiológicos, datados de 1960, revelaram que a população africana raramente sofre de constipação intestinal e de câncer do intestino, estando relacionado ao elevado consumo de fibras (CUMMINGS; EDMOND; MAGEE, 2004).

\section{Conclusões}

De acordo com os resultados da avaliação sensorial, as amostras Controle e as adicionadas de FOS tiveram melhor aceitação. A amostra contendo somente FOS foi a que mais se aproximou do produto tradicional, não diferindo significativamente quanto à aceitação global. Esse resultado permite concluir que o FOS é um ingrediente promissor no desenvolvimento de produtos ricos em fibras, sendo possível produzir sorvete com aceitação sensorial semelhante ao tradicional, agregando valor nutricional ao produto.

\section{Referências bibliográficas}

AGÊNCIA NACIONAL DE VIGILÂNCIA SANITÁRIA. ANVISA. (Brasil). Alimentos. Comissões e Grupos de Trabalho. Comissão Tecnocientífica de Assessoramento em Alimentos Funcionais e Novos Alimentos. Alimentos com Alegações de Propriedades Funcionais e ou de Saúde, Novos Alimentos/ Ingredientes, Substâncias Bioativas e Probiótios. Disponível em: <http:www. anvisa.gov.br/alimentos/comissões/tecno_lista_alega.htm $>$. Acesso em: 13 abr. 2008. (Atualizado em agosto/2007. Lista de alegações de propriedade funcional aprovadas).

. Informe Técnico $n^{\circ} 9$, de 21 de maio de 2004. Orientação para utilização, em rótulos de alimentos, de alegação de propriedades funcionais de nutrientes com funções plenamente reconhecidas pela comunidade científica (item 3.3 da Resolução ANVS/MS no18/99). Disponível em: <http://www.anvisa.gov.br/alimentos/ informes/09_210504.htm>. Acesso em: 13 abr. 2008.

Resolução RDC n 359, de 23 de dezembro de 2003. Aprova Regulamento Técnico de Porções de Alimentos Embalados para Fins de Rotulagem Nutricional. Diário Oficial da República Federativa do Brasil, Brasília, 26 dez. 2003. Disponível em: $<$ http://e-legis.anvisa.gov.br/leisref/public/showAct.php?id=9058>. Acesso em: 13 abr. 2008.

ASSOCIATION OF OFFICIAL ANALYTICAL CHEMISTS. Official Methods of Analysis of the AOAC. $16^{\text {th }}$ ed. Washington (DC): AOAC, 2002.

BEHRENS, J. H.; SILVA, M. A. A. P.; WAKELING, I. N. Avaliação da aceitação de vinhos brancos varietais brasileiros através de testes sensoriais afetivos e técnica multivariada de Mapa de Preferência interno. Ciência e Tecnologia de Alimentos, v. 19. n. 2, p. 214-220, 1999.

COZZOLINO, S. M. F. Biodisponibilidade de nutrientes. Barueri, SP: Editora Manole, 2005. 878 p.

CUMmingS, J. H.; EDMOND, L. M.; MAGEE, E. A. Dietary carbohydrates and health: do we still need the fibre concept? Clinical Nutrition Supplements, v. 1, n. 2, p. 5-17, 2004.

FERREIRA, C. L. L. F. Tecnologia para produtos lácteos funcionais: probióticos. In: PORTUGAL, J. A. B.; CASTRO, M. C. D.; SILVA, P. H. F.; SAVINO, A. C.; NEVES, B. S.; ARCURI, E. F. O agronegócio do leite e os alimentos lácteos funcionais. Juiz de Fora: EPAMIG - Centro Tecnológico- ILCT, 2001. Cap.4, p. $183-203$
FERREIRA, L. G.; BURINI, R. C.; MAIA, A. F. Dietas vegetarianas e desempenho esportivo. Revista de Nutrição, v. 19, n. 4, p. 469-477, 2006.

FRANCO, G. Tabela de composição química dos alimentos. 9 ed. Rio de Janeiro: Atheneu, 1999.

GERMAN, B. et al. The development of functional foods: lessons from the gut. Trends in Biotechnology, v. 17, n. 12, p. 492-499, 1999.

GRANADA, G. et al. Caracterização de granolas comerciais. Ciência e Tecnologia de Alimentos, v. 23, n. 1, p. 87-91, 2003.

GRIZARD, D.; DALLE, M.; BARTHOMEUF, C. Changes in insulin and cortiosterone levels may partly mediate the hypolipidemic effect of guar gum and low-molecular weight pectin in rats. Nutrition Research, v. 21, n. 8, p. 1185-1190, 2001.

GUILLON, F.; CHAMP, M. Strutural and physical properties of dietary fibres, and consequences of processing on human physiology. Food Research International, v. 33, n. 3, p. 233-245, 2000.

HARDY, G. Nutraceuticals and functional foods: Introduction and meaning. Nutrition, v. 16, n. 7, p. 688-689, 2000.

NATIONAL ACADEMY OF SCIENCES. Institute Of Medicine. Food and Nutrition Board. Dietary references intakes for energy, carbohydrate, fiber, fat, fatty acids, cholesterol, protein and amino acids (Macronutrients). Washington, DC: The National Academy Press, 2002.

IZZO, M.; NINESS, K. Formulating nutrition bars with inulin and oligofructose. Cereal Foods World, v. 46, n. 3, p. 102-106, 2001

PINHEIRO, A. B. V. et al. Tabela para avalição do consumo alimentar em medidas caseiras. 5 ed. São Paulo: Atheneu, 2005. 131 p.

QI, L. et al. Dietary Fibers and Glycemic Load, Obesity, and Plasma Adiponectin Levels in Women With Type 2 Diabetes. Diabetes Care, v. 29, p. 1501-1505, 2006.

REIS, C. R.; MINIM, V. P. R. Testes de aceitação. In: MINIM, V. P. R. (Ed.). Análise sensorial: estudos com consumidores. Viçosa: Editora UFV, 2006. Cap. 3, p. 67-83.

REIS, C. R. et al. Mapa de Preferência. In: MINIM, V. P. R (Ed). Análise sensorial: estudos com consumidores. Viçosa: Editora UFV, 2006. Cap. 5, p. 111-126.

RIBEIRO, P. et al. Tabelas de composição química de alimentos: análise comparativa com resultados laboratoriais. Revista de Saúde Pública, v. 37, n. 2, p. 216-225, 2003.

RODRÍGUEZ, M. B. S.; MEGÍAS, S. M.; BAENA, B. M. Alimentos Funcionales y Nutrición Óptima. ¿CERCA O LEJOS? Revista Española de Salud Pública, v. 77, n. 3, p. 317-331, 2003.

RODRÍGUEZ, R. et al. Dietary fibre from vegetable products as source of functional ingredients. Trends in Food Science \& Technology, v. 17, n. 1, p. 3-15, 2006.

SARTORELLI, D. S.; FRANCO, L. J. Tendências do diabetes mellitus no Brasil: o papel da transição nutricional. Cadernos de Saúde Pública, v. 19, n. 1, p. 29-36, 2003.

SGARBIERI, V. C.; PACHECO, M. T. B. Revisão: Alimentos Funcionais Fisiológicos. Brazilian Journal of Food Technology, v. 2, n. 2, p. 7-19, 1999.

SAS - STATISTICAL ANALYSIS SYSTEM. Versión 8.0. Cary, North Carolina: The SAS Institute, 1999.

SWADLING, I. The regulation and marketing of functional foods worldwide. Food Info, p. 4-5, 2001.

URGELL, M. R.; ORLEÁNS, A. S.; SEUMA, M. R. P. La importancia de los ingredientes funcionales en las leches y cereales infantiles. Nutricion Hospatalaria, v. 20, n. 2, p. 135-146, 2005. 